Hansen AK, Brooks DJ, Borghammer P. MAO-B Inhibitors Do Not Block In Vivo Flortaucipir ([ $\left.\left.{ }^{18} \mathrm{~F}\right]-\mathrm{AV}-1451\right)$ Binding. Molecular Imaging and Biology (2017)

DOI link

https://doi.org/10.1007/s11307-017-1143-1

ePrints link

http://eprint.ncl.ac.uk/pub details2.aspx?pub id=243049

Date deposited

$16 / 01 / 2018$

Embargo release date

$10 / 11 / 2018$

Copyright

The final publication is available at Springer via https://doi.org/10.1007/s11307-017-1143-1 


\section{MAO-B inhibitors do not block in vivo flortaucipir ([18F]-AV-1451) binding}

Allan K Hansen ${ }^{1}$, David J Brooks ${ }^{1,2,3}$, Per Borghammer ${ }^{1}$

${ }^{1}$ Dept of Nuclear Medicine \& PET-Centre, Institute of Clinical Medicine, Aarhus University, Denmark

${ }^{2}$ Division of Neuroscience, Dept of Medicine, Imperial College London, UK

${ }^{3}$ Division of Neuroscience, Newcastle University, UK

Corresponding author: Allan K Hansen, MD, PhD-student

PET-Centre, Aarhus University Hospital

Norrebrogade 44, bld. $10 \mathrm{G}$

DK-8000 Aarhus C

M: +4540116831

E: ah@clin.au.dk

Word count:

3130

Financial support:

This study was financed through a grant from the Lundbeck Foundation.

Running head:

Flortaucipir and MAO-B inhibition 


\section{Abstract:}

\section{PURPOSE:}

Recent evidence suggests that the tau radiotracer $\left[{ }^{18} \mathrm{~F}\right]-\mathrm{THK}-5351$ displays high affinity for the monoamine oxidase type B (MAO-B) enzyme. Utilizing another tau-tracer, flortaucipir ([ $\left.\left.{ }^{18} \mathrm{~F}\right]-\mathrm{AV}-1451\right)$, we previously reported that non-demented Parkinson's disease patients show off-target binding in subcortical structures, but no appreciable cortical uptake. However, $59 \%$ of these patients were receiving MAO-B inhibitors at the time of their scan. Here, we retrospectively investigated if MAO-B inhibitors in clinical doses affect flortaucipir binding.

\section{PROCEDURES:}

We compared the standard uptake values of flortaucipir at regional and voxel levels in Parkinson's disease patients who received MAO-B inhibitors with those who did not.

\section{RESULTS:}

Sixteen of 27 Parkinson's disease patients received MAO-B inhibitors at the time of scan. We found no significant flortaucipir uptake differences between the groups at voxel or regional levels.

\section{CONCLUSION:}

Use of MAO-B inhibitors at pharmaceutical levels did not significantly affect flortaucipir binding. Thus, MAO-B does not appear to be a significant binding target of flortaucipir.

Key words:

Tau, neuroimaging, flortaucipir, MAO-B 


\section{Introduction}

Flortaucipir, also known as $\left[{ }^{18} \mathrm{~F}\right]-\mathrm{AV}-1451$ or $\left[{ }^{18} \mathrm{~F}\right]-\mathrm{T} 807$, has been shown to detect cortical tau pathology in the form of neurofibrillary tangles, in increasing amounts over the spectrum from healthy aging, mild cognitive impairment, and Alzheimer's disease[1-4]. The tracer, however, also displays age-related offtarget binding of unclear origin in the basal ganglia, and to neuromelanin rich cells of the substantia nigra and retina[5-7]. Another tau-tracer, $\left[{ }^{18} \mathrm{~F}\right]-$ THK-5351, with a similar uptake pattern to flortaucipir at a macroscopic level, was recently shown to be a substrate for monoamine oxidase type B (MAO-B) in vivo and in autoradiography and its binding was blocked by the MAO-B inhibitor selegiline[8]. Interestingly, positron emission tomography (PET) scans with the MAO-B marker $\left[{ }^{11} \mathrm{C}\right]$-deprenyl[9] show similarities with $\left[{ }^{18} \mathrm{~F}\right]-$ THK-5351 and flortaucipir PET scans. Further, brain MAO-B is known to increase with age[10]. It is, therefore, conceivable that MAO-B is also a target of flortaucipir.

We have previously reported on the flortaucipir binding pattern of a group of patients with Parkinson's disease[5, 11]. 59\% of these tau negative patients who had flortaucipir PET had received MAO-B inhibitors within 24 hours of their scan, as we were unaware at that time of the possible influence of MAO-B activity on flortaucipir binding. With the purpose of investigating the validity of our previous studies, and for future reference in clinical use, we retrospectively investigated if MAO-B inhibitor use in clinical doses affects their flortaucipir binding.

\section{Materials and Methods}

Twenty-seven Parkinson's disease patients who have been previously described $[5,11]$ were retrospectively stratified based on their MAO-B inhibitor use based on their lists of medication at the time of the flortaucipir scan[5]. The study was approved by the regional ethics committee and all subjects signed an informed consent form. Scan coregistration, volume of interest definition, and data extraction were performed with PMOD software (PMOD, Zürich, Switzerland), as previously described[5]. 
Regional statistical analysis was performed with Stata 13.1 (StataCorp LP, Texas). Voxel wise analyses were performed with SPM12 (http://www.fil.ion.ucl.ac.uk/spm/), using a linear regression model with standardized uptake values as the dependent variable and group and age as independent variables. Familywise error corrections were used for voxel wise analyses. Continuous and categorical variables were interrogated using Student's t-test and Fisher's exact test, respectively. P-values $<0.05$ were considered statistically significant.

\section{Results}

Six of 27 Parkinson's disease patients reported taking selegiline (one $5 \mathrm{mg}$, four $10 \mathrm{mg}$, and one $15 \mathrm{mg}$ per day) and ten other patients reported taking rasagiline (all $1 \mathrm{mg}$ per day) on the day of scan. See TABLE 1 for demographics.

No significant between group differences in mean flortaucipir uptake were seen in regional (FIGURE 1) or voxel wise analyses (FIGURE 2). A more liberal, explorative approach with simple inspection of t-maps revealed no meaningful trends in group differences.

\section{Discussion}

Our results suggest that flortaucipir PET is not significantly affected by oral ingestion of MAO-B inhibitors at clinically prescribed doses. It, therefore, seems unlikely that MAO-B binding is contributing to the basal ganglia signal seen in flortaucipir PET images. However, we cannot rule out non-competitive binding of flortaucipir to a different binding site on MAO-B, which may be unaffected by the MAO-B inhibitors in clinical use.

In an early paper on flortaucipir (then known as $\left[{ }^{18} \mathrm{~F}\right]-\mathrm{T} 807$ ), Xia and colleagues[12] found that "no inhibition was seen for MAO-A and MAO-B at a 1- $\mu M$ concentration". This was specifically tested, as the group of compounds the tracer belongs to, the carbazoles, was found to contain potent MAO inhibitors. Later, in a conference presentation, Vermeiren and colleagues reported affinity of flortaucipir for MAO-A $\left(\mathrm{K}_{\mathrm{D}} \approx 1.5 \mathrm{nM}\right)$ 
and MAO-B $\left(\mathrm{K}_{\mathrm{D}} \approx 30 \mathrm{nM}\right)[13]$, though this has not to our knowledge been published in detail. Several unsuccessful attempts have been made to identify other targets using autoradiography[6, 7, 14, 15].

Following the recent paper by Kok $\mathrm{Ng}$ and colleagues describing up to a $50 \%$ reduction in $\left[{ }^{18} \mathrm{~F}\right]-\mathrm{THK}-5351$ signal following selegiline administration, we felt it was appropriate to revisit the potential for a significant effect of MAO-B inhibitor use on flortaucipir PET. A characteristic pattern of heterogenous distribution with relatively higher signal in the striatum, globus pallidus, and thalamus is shared between flortaucipir[2, 16], $\left[{ }^{18} \mathrm{~F}\right]-$ THK-5351[8] and $\left[{ }^{18} \mathrm{~F}\right]$-labeled selegiline $\left(\left[{ }^{18} \mathrm{~F}\right]\right.$-fluorodeprenyl-D)[17]. Further, the binding of these three tracers all extend to include cortical areas in Alzheimer's disease (flortaucipir[1], $\left[{ }^{18} \mathrm{~F}\right]-\mathrm{THK}-5351[18]$ and $\left[{ }^{11} \mathrm{C}\right]$-labelled selegiline $\left(\left[{ }^{11} \mathrm{C}\right]\right.$-deuterium-L-deprenyl)[9]). One notable exception to this pattern is that flortaucipir has additional off-target binding in the substantia nigra[7], presumed to be neuromelanin, and also to the choroid plexus[19]. The similar brain distributions of flortaucipir uptake and MAO-B activity and the age dependency of both suggests the possibility of significant MAO-B binding by flortaucipir [10]. Also, the paradoxical pattern of flortaucipir PET in progressive supranuclear palsy patients, with an absence of binding in cortex but increased binding in the basal ganglia compared with healthy controls, could be compatible with MAO-B binding of this agent $[6,16,20-22]$. The observations in progressive supranuclear palsy may be explained by increased amounts of MAO-B from associated gliosis, rather than binding to the 4-repeat tau present. The linear tau seen in progressive supranuclear palsy has been autoradiographically shown to have a low affinity for flortaucipir as compared to the mixed 3 and 4 repeat paired helical tau of Alzheimer's disease[7, 14]. Further, the unexplained increased flortaucipir binding in the putamen of multiple system atrophy[23], could also be attributable to concomitant gliosis and raised MAO-B. Indeed, Shcherbinin and colleagues reported findings suggesting "either additional off-target binding in the putamen or a second binding site with different kinetics" [24]. We have previously reported reduced offtarget flortaucipir binding in the substantia nigra of Parkinson's disease[5], compatible with a decrease in neuromelanin content[25]. While similar pigment-like compounds increasing with age have been described 
in the basal ganglia[26], Passamonti and colleagues found no neuromelanin-containing cells in the basal ganglia in patients or controls with relatively increased in vivo flortaucipir PET binding in the same area[6].

If MAO-B is a significant target of flortaucipir at levels used for in vivo PET, it diminishes the tracer's value as a marker of tau and an outcome measure in pharmaceutical trials. Further, as brain MAO-B (and $-\mathrm{A})$ is reduced by smoking[27], present smoking status would have to be recorded for clinical use. As MAO-B appears to be a significant target of $\left[{ }^{18} \mathrm{~F}\right]-$ THK-5351, while flortaucipir does not, we expect higher affinity between $\left[{ }^{18} \mathrm{~F}\right]-\mathrm{THK}-5351$ and MAO-B, than between flortaucipir and MAO-B. However, we are not aware of any publication reporting results of MAO-B to $\left[{ }^{18} \mathrm{~F}\right]-\mathrm{THK}-5351$ binding assays, which would allow such comparison. While a relative difference in affinity of the two tau tracers for MAO-B would explain the findings of the present study, the off-target binding of flortaucipir in the basal ganglia still remains to be elucidated. The importance of identifying this target is underlined by the example of $\left[{ }^{18} \mathrm{~F}\right]-\mathrm{THK}-5351$, i.e. the off-target may co-localize with the primary target of tau in neurofibrillary tangles.

According to Vermeiren and colleagues[13] and the investigator's brochure, flortaucipir displays higher affinity for MAO-A than MAO-B[13]. This affinity for MAO-A may be less problematic as MAO-A occurs in lower concentration, and does not show age-dependent increases[10]. Further, the increase of MAO activity from astrocytes observed in Alzheimer's disease, appear to be due to increased MAO-B activity alone[28]. To our knowledge, no attempts have been made to block flortaucipir signal using the MAO-A inhibitor clorgyline. Clorgyline can, however, be used as a PET-tracer, and shows a pattern of high binding in the thalamus and occipital cortex, intermediate values in basal ganglia, frontal, and temporal cortices and little binding in the cerebellum[29, 30]. Another MAO-A tracer, $\left[{ }^{11} \mathrm{C}\right]$-harmine[31], displays a similar pattern[32]. Hence, the binding pattern of these MAO-A tracers does not appear to match the pattern shown by flortaucipir.

This study has several limitations. First, we did not have paired blocked and unblocked patient data. However, Kok Ng and colleagues showed robust $\left[{ }^{18} \mathrm{~F}\right]-\mathrm{THK}-5351$ reductions of $>50 \%$ in the thalamus and 
basal ganglia after administration of clinical selegiline doses. The comparable $95 \%$ confidence intervals of mean thalamic flortaucipir decrease following MAO-B inhibitor administration in our study was [-11.2\%; 24.0\%]. Second, we did not have an exact record of when the test subjects received their last dose of MAO$\mathrm{B}$ inhibitor prior to the scan or for how long they had received MAO-B inhibitors. Using $\left[{ }^{11} \mathrm{C}\right] \mathrm{L}$-deprenyl, Fowler and colleagues demonstrated that one week of treatment with selegiline in clinical doses (10 mg per day) blocks more than $95 \%$ of binding to MAO-B. This corresponds to a $70-80 \%$ reduction in overall brain SUV, due to increased tracer availability from blocking elsewhere. Following selegiline withdrawal, MAO-B recovered with a half-time of 40 days[33, 34]. Consequently, the impact of a patient having missed a single or even two doses before scan would be negligible. Third, the use of chronic administration in our study, rather than acute treatment as in Kok Ng et al., may cause unforeseen effects, including compensatory regulation of the MAO-A and -B enzymes, which could ultimately affect the results. Fourth, two different MAO-B inhibitors with different structures and metabolites were used. Considering the large effect size described by Kok and colleagues, we would expect to see at least a bimodal distribution in the MAO-B inhibitor group, even if only selegiline and not rasagiline was an effective blocker of flortaucipir binding. Finally, as none of our Parkinson's disease patients appeared to be tau-positive, we were unable to investigate if the specific binding from areas with neurofibrillary tangles was blockable with MAO-B inhibitors[9, 35].

\section{Conclusion}

Several observations from human PET studies suggest that MAO-B as well as tau could be a target of flortaucipir binding. However, after retrospectively comparing Parkinson's disease patients receiving clinical doses of MAO-B inhibitors with those who did not, we were unable to detect any significant effect of MAOB inhibition on flortaucipir uptake. Further studies are needed to investigate the origin of the off-target binding of flortaucipir. 


\section{Conflict of Interest Statement}

Allan K Hansen: $\quad$ None

Per Borghammer: Consultancy with F. Hoffman-La Roche and has received grant support from Lundbeck foundation, Jascha foundation, the Danish Parkinson Foundation, and Aarhus University (Ph.D. salaries)

David J Brooks: Has served on the Neuroscience and Mental Health Board of the Medical Research Council, UK; is associate editor of Brain and serves or has served as an editorial board member for Parkinsonism and Related Disorders, Journal of Parkinson's Disease, Journal of Neural Transmission, Movement Disorders, Journal of Neurology, Neurosurgery and Psychiatry, and Synapse; he has received honoraria from TEVA, Orion Pharma, GlaxoSmithKline, Genentech and Elan; holds stock options and is a consultant for GE Healthcare; receives research support from the EU FP7 program, Alzheimer's Research Trust UK, Parkinson's UK, the Medical Research Council, UK, Danish Council for Independent Research, and the Lundbeck Foundation.

\section{Acknowledgements}

We thank Avid Radiopharmaceuticals, Inc. for providing precursor for the radiochemical tracer synthesis.

\section{References}

1. Johnson KA, Schultz A, Betensky RA, et al (2016) Tau positron emission tomographic imaging in aging and early Alzheimer disease. Ann Neurol 79:110-9.

2. Schöll M, Lockhart SN, Schonhaut DR, et al (2016) PET Imaging of Tau Deposition in the Aging Human Brain. Neuron 89:971-982.

3. Cho H, Choi JY, Hwang MS, et al (2016) Tau PET in Alzheimer disease and mild cognitive impairment. Neurology 87:375-83. 
4. Pontecorvo MJ, Devous MD, Navitsky M, et al (2017) Relationships between flortaucipir PET tau binding and amyloid burden, clinical diagnosis, age and cognition. Brain 140:748-763.

5. Hansen AK, Knudsen K, Lillethorup TP, et al (2016) In vivo imaging of neuromelanin in Parkinson's disease using 18F-AV-1451 PET. Brain 139:2039-49.

6. Passamonti L, Vázquez Rodríguez P, Hong YT, et al (2017) 18F-AV-1451 positron emission tomography in Alzheimer's disease and progressive supranuclear palsy. Brain 140:781-791.

7. Marquié $M$, Normandin MD, Vanderburg CR, et al (2015) Validating novel tau positron emission tomography tracer [F-18]-AV-1451 (T807) on postmortem brain tissue. Ann Neurol 78:787-800.

8. Ng KP, Pascoal TA, Mathotaarachchi S, et al (2017) Monoamine oxidase B inhibitor, selegiline, reduces 18F-THK5351 uptake in the human brain. Alzheimers Res Ther 9:25.

9. Carter SF, Scholl M, Almkvist O, et al (2012) Evidence for Astrocytosis in Prodromal Alzheimer Disease Provided by 11C-Deuterium-L-Deprenyl: A Multitracer PET Paradigm Combining 11CPittsburgh Compound B and 18F-FDG. J Nucl Med 53:37-46.

10. Tong J, Meyer JH, Furukawa Y, et al (2013) Distribution of monoamine oxidase proteins in human brain: implications for brain imaging studies. J Cereb Blood Flow Metab 33:863-71.

11. Hansen AK, Damholdt MF, Fedorova TD, et al (2017) In Vivo cortical tau in Parkinson's disease using 18F-AV-1451 positron emission tomography. Mov Disord 32:922-927.

12. Xia CF, Arteaga J, Chen G, et al (2013) [(18)F]T807, a novel tau positron emission tomography imaging agent for Alzheimer's disease. Alzheimers Dement 9:666-76.

13. Vermeiren C, Mercier J, Viot D, et al (2015) T807, a reported selective tau tracer, binds with nanomolar affinity to monoamine oxidase a. Alzheimer's Dement 11:P283. 
14. Lowe VJ, Curran G, Fang P, et al (2016) An autoradiographic evaluation of AV-1451 Tau PET in dementia. Acta Neuropathol Commun 4:58.

15. Marquié M, Normandin MD, Meltzer AC, et al (2017) Pathological correlations of [F-18]-AV-1451 imaging in non-alzheimer tauopathies. Ann Neurol 81:117-128.

16. Smith R, Schöll M, Honer M, et al (2017) Tau neuropathology correlates with FDG-PET, but not AV1451-PET, in progressive supranuclear palsy. Acta Neuropathol 133:149-151.

17. Nag S, Fazio P, Lehmann L, et al (2016) In Vivo and In Vitro Characterization of a Novel MAO-B Inhibitor Radioligand, 18F-Labeled Deuterated Fluorodeprenyl. J Nucl Med 57:315-20.

18. Lockhart SN, Baker SL, Okamura N, et al (2016) Dynamic PET measures of tau accumulation in cognitively normal older adults and Alzheimer's disease patients measured using [18F] THK-5351. PLoS One 11:1-16.

19. Ikonomovic MD, Abrahamson EE, Price JC, et al (2016) [F-18]AV-1451 positron emission tomography retention in choroid plexus: More than "off-target" binding. Ann Neurol 80:307-8.

20. Cho H, Choi JY, Hwang MS, et al (2017) Subcortical (18) F-AV-1451 binding patterns in progressive supranuclear palsy. Mov Disord 32:134-140.

21. Whitwell JL, Lowe VJ, Tosakulwong N, et al (2017) [(18) F]AV-1451 tau positron emission tomography in progressive supranuclear palsy. Mov Disord 32:124-133.

22. Smith R, Schain M, Nilsson C, et al (2017) Increased basal ganglia binding of (18) F-AV-1451 in patients with progressive supranuclear palsy. Mov Disord 32:108-114.

23. Cho H, Choi JY, Lee SH, et al (2017) (18) F-AV-1451 binds to putamen in multiple system atrophy. Mov Disord 32:171-173. 
24. Shcherbinin S, Schwarz AJ, Joshi A, et al (2016) Kinetics of the Tau PET Tracer 18F-AV-1451 (T807) in Subjects with Normal Cognitive Function, Mild Cognitive Impairment, and Alzheimer Disease. J Nucl Med 57:1535-1542.

25. Zecca L, Fariello R, Riederer P, et al (2002) The absolute concentration of nigral neuromelanin, assayed by a new sensitive method, increases throughout the life and is dramatically decreased in Parkinson's disease. FEBS Lett 510:216-220.

26. Zecca L, Bellei C, Costi P, et al (2008) New melanic pigments in the human brain that accumulate in aging and block environmental toxic metals. Proc Natl Acad Sci U S A 105:17567-17572.

27. Fowler JS, Logan J, Wang G-J, Volkow ND (2003) Monoamine oxidase and cigarette smoking. Neurotoxicology 24:75-82.

28. Nakamura S, Kawamata T, Akiguchi I, et al (1990) Expression of monoamine oxidase B activity in astrocytes of senile plaques. Acta Neuropathol 80:419-425.

29. Fowler JS, Volkow ND, Wang GJ, et al (1996) Brain monoamine oxidase A inhibition in cigarette smokers. Proc Natl Acad Sci U S A 93:14065-9.

30. Fowler JS, MacGregor RR, Wolf AP, et al (1987) Mapping human brain monoamine oxidase A and B with 11C-labeled suicide inactivators and PET. Science 235:481-5.

31. Jensen SB, Olsen AK, Pedersen K, Cumming P (2006) Effect of monoamine oxidase inhibition on amphetamine-evoked changes in dopamine receptor availability in the living pig: A dual tracer PET study with [11C]harmine and [11C]raclopride. Synapse 59:427-434.

32. Ginovart N, Meyer JH, Boovariwala A, et al (2006) Positron emission tomography quantification of [11C]-harmine binding to monoamine oxidase-A in the human brain. J Cereb Blood Flow Metab 26:330-44. 
33. Fowler JS, Volkow ND, Logan J, et al (1994) Slow recovery of human brain MAO B after L-deprenyl (Selegeline) withdrawal. Synapse 18:86-93.

34. Arnett CD, Fowler JS, MacGregor RR, et al (1987) Turnover of brain monoamine oxidase measured in vivo by positron emission tomography using L-[11C]deprenyl. J Neurochem 49:522-7.

35. Lemoine L, Saint-Aubert L, Nennesmo I, et al (2017) Cortical laminar tau deposits and activated astrocytes in Alzheimer's disease visualised by 3H-THK5117 and 3H-deprenyl autoradiography. Sci Rep 7:45496. 


\section{Figure Legends}

FIGURE 1. Key regional standardized uptake values (SUV) from all subjects, no age correction. Monoamine oxidase type B (MAO-B).

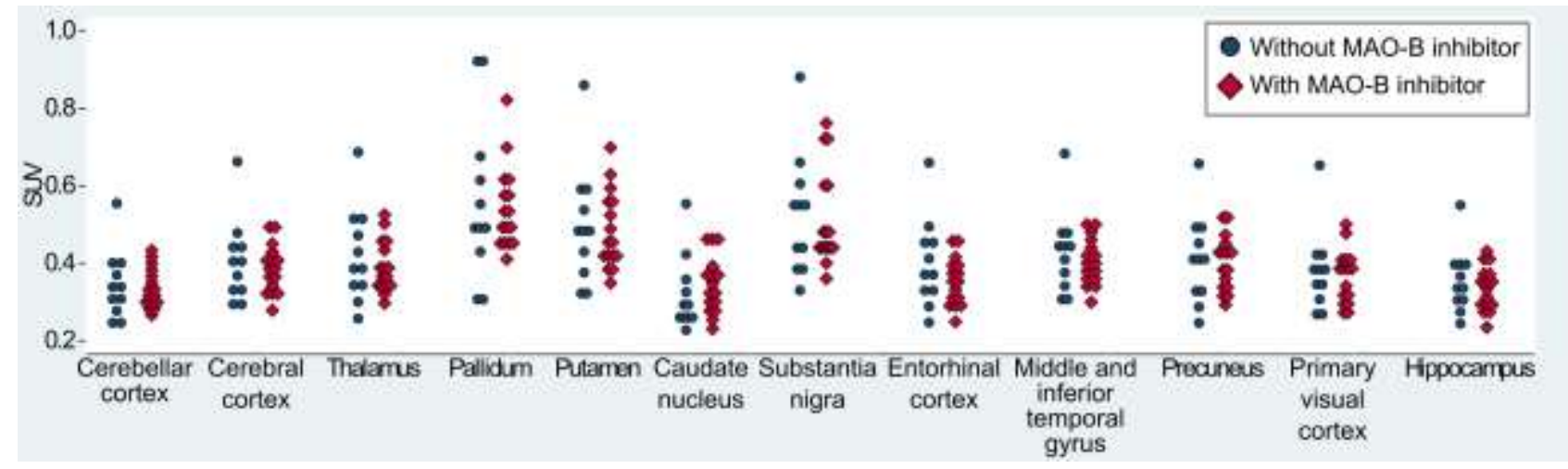


FIGURE 2. Mean standardized uptake values (SUV) of the two groups, corrected to age 70 years old. 1:

Caudate nucleus. 2: Putamen. 3: Choroid plexus. 4: Pallidum. Monoamine oxidase type B (MAO-B).

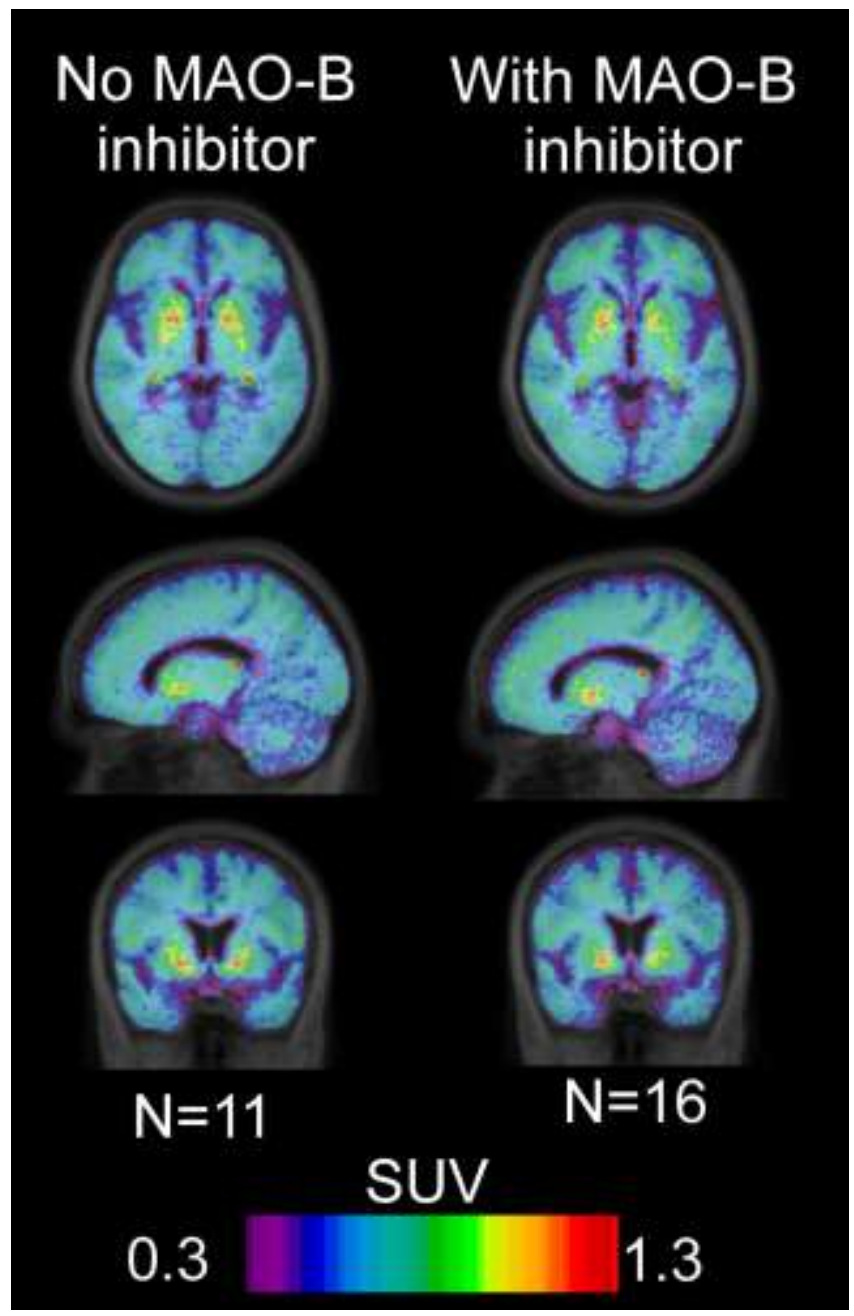


TABLE 1 - Demographics

\begin{tabular}{lcc}
\hline Parkinson's disease group: & No MAO-B inhibitor & With MAO-B inhibitor \\
\hline Study group, N & 11 & 16 \\
PD-MCI/nonMCI & $5 / 6$ & $4 / 12$ \\
\hline Age & $70.6 \pm 8.3$ & $66.7 \pm 5.0$ \\
Female/Male & $2 / 9$ & $2 / 14$ \\
Smoker: Never/Previous & $8 / 3$ & $10 / 6$ \\
Montreal Cognitive Assesment & $26.1 \pm 2.4$ & $26.9 \pm 2.5$ \\
Mini-Mental State Examination & $28.1 \pm 1.4$ & $28.6 \pm 1.5$ \\
\hline Unified Parkinson's Disease Rating Scale, Part 3 & $21.4 \pm 12.7$ & $24.5 \pm 13.9$ \\
\hline Hoehn \& Yahr stage & $2.0 \pm 0.6$ & $2.3 \pm 0.6$ \\
\hline Disease duration, years & $5.4 \pm 3.7$ & $5.5 \pm 3.7$
\end{tabular}

Mean \pm standard deviation. No significant differences as tested with Student's t-test or Fisher's exact test.

Parkinson's Disease with Mild Cognitive Impairment (PD-MCI). 